\title{
Enlarged analogues of uniconazole, new azole containing inhibitors of ABA 8'-hydroxylase CYP707A
}

Yasushi Todoroki, ${ }^{\mathrm{a} *}$ Hikaru Aoyama, ${ }^{\mathrm{a}}$ Saori Hiramatsu, ${ }^{\mathrm{a}}$ Minaho Shirakura, ${ }^{\mathrm{a}}$ Hataitip

Nimitkeatkai, ${ }^{b}$ Satoru Kondo, ${ }^{b}$ Kotomi Ueno,,${ }^{c, \dagger}$ Masaharu Mizutani, ${ }^{d, \ddagger}$ Nobuhiro Hirai ${ }^{\mathrm{e}}$

${ }^{a}$ Department of Applied Biological Chemistry, Faculty of Agriculture, Shizuoka University, Shizuoka 422-8529, Japan

${ }^{b}$ Division of Bioresource Science, Graduate School of Horticulture, Chiba University, Matsudo 271-8510, Japan

${ }^{c}$ The United Graduate School of Agricultural Science, Gifu University, Gifu 501-1193, Japan

${ }^{d}$ Institute for Chemical Research, Kyoto University, Uji, Kyoto 611-0011, Japan

${ }^{e}$ Division of Environmental Science and Technology, Graduate School of Agriculture, Kyoto University, Kyoto 606-8501, Japan

*Corresponding author. Phone/Fax:+81-54-238-4871. E-mail: aytodor@agr.shizuoka.ac.jp

†Present address: Graduate School of Agricultural and Life Sciences, The University of Tokyo, Tokyo 113-8657, Japan.

${ }^{\ddagger}$ Present address: Graduate School of Agricultural Science, Kobe University, Kobe 657-8501, Japan. 
Abstract - We enlarged the uniconazole (UNI) molecule to find a specific inhibitor of abscisic acid (ABA) 8'-hydroxylase, and synthesized various UNI derivatives that were substituted with hydrophilic and hydrophobic groups at the 4-chlorine of the phenyl group of UNI using click chemistry. Considering its potency in ABA 8'-hydroxylase inhibition, its small effect on seedling growth, and its ease of application, UT4, the UNI derivative containing the $\mathrm{C}_{4}$ alkyltriazole, was the best candidate for a highly selective inhibitor of ABA 8'-hydroxylase. 
Abscisic acid (ABA) is a plant hormone involved in stress tolerance, stomatal closure, seed dormancy, and other physiological events. ${ }^{1-4}$ The endogenous levels of ABA in plants are cooperatively controlled by biosynthesis, transportation, and catabolic inactivation in response to environmental changes. ${ }^{1-4}$ A natural or artificial chemical that perturbs this highly controlled system is promising not only as a chemical probe for the mechanism of ABA action, ${ }^{5}$ but also because of its potential use in agriculture and horticulture. Although ABA is registered as a farm chemical (plant growth regulator), its practical use has been limited mainly due to its weak effect in field trials, ${ }^{6}$ which is considered to be due to its rapid inactivation through biodegradation. Catabolic inactivation of ABA is mainly controlled by $\mathrm{ABA}$ 8'-hydroxylase, which is the cytochrome P450 catalyzing the C8'-hydroxylation of ABA into 8'-hydroxy-ABA and its more stable tautomer, phaseic acid, which has much lower hormonal activity than ABA (Figure 1). ${ }^{4}$ ABA 8'-hydroxylase was identified as CYP707A1-4 in the model plant Arabidopsis thaliana in 2004,,$^{7,8}$ and since then many CYP707A isozymes have been found in various plants. ${ }^{9-12}$ Gene knockdown and overexpression studies suggest that ABA 8'-hydroxylase is a key enzyme for controlling ABA concentration during water deficit stress or dormancy maintenance and breaking. ${ }^{13,14}$

To chemically control ABA 8'-hydroxylation in plants, we are developing ABA analogues that function as competitive inhibitors against ABA 8'-hydroxylase. ${ }^{15-17}$ AHI4, which was developed in a previous study, requires a very high concentration $(>400 \mu \mathrm{M})$ to confer drought tolerance by its exogenous application. ${ }^{17}$ On the other hand, uniconazole (UNI), which is a well-known plant retardant developed in the 1980s, ${ }^{18,19}$ strongly inhibits ABA 8'-hydroxylase both in vitro and in vivo. ${ }^{20-22}$ Saito et al. reported that UNI induces drought tolerance in Arabidopsis plants at $50 \mu \mathrm{M}^{21}$ The main site of action of UNI is suggested to be ent-kaurene oxidase (CYP701A), ${ }^{23}$ which catalyzes the three-step oxidation of ent-kaurene to ent-kaurenoic acid, a biosynthetic precursor of the plant hormone gibberellin (Figure 1). Thus, UNI has been used as a chemical tool inhibiting gibberellin biosynthesis. UNI also inhibits brassinosteroid 
biosynthesis $^{24,25}$ and alters the level of other plant hormones, such as auxins, cytokinins, and ethylene. $^{26}$ Thus, we cannot use UNI in expectation of only inhibiting ABA catabolism, in spite of its strong effect on ABA levels.

Azole-type P450 inhibitors including UNI bind to the target P450 active site by both coordinating to the heme-iron atom and interacting with surrounding protein residues. Because heme coordination is a common property of azole-containing inhibitors, their affinity and specificity for individual P450 enzymes depend on structural properties other than the azole group. UNI may be small and flexible enough to embed itself into the various substrate-binding pockets. This led us to the possibility that enlarging or conformationally freezing the UNI molecule would result in a narrow inhibition spectrum. For this research, we focused on enlarging the molecule.

Although the crystal structures of P450s indicate an overall similarity in structural folding in spite of limited sequence identity, regions responsible for substrate binding differ substantially. These regions correspond to the substrate recognition sites, SRS1- $6 .{ }^{27}$ In particular, SRS1-3 and 6, which are far from the heme in the active site, are structurally quite variable and are involved significantly in substrate specificity. In the case of UNI, the portion of the molecule far from the triazole interacting with the heme is the 4-chlorine of the phenyl ring, which might interact with SRS1-3 and 6. Thus, we selected C4 of the phenyl in UNI as a scaffold for enlarging the UNI molecule. This paper describes the synthesis, CYP707A-inhibitory properties and biological activities of the enlarged UNI analogues, UT.

We used click chemistry ${ }^{28}$, by which a variety of compounds are easily prepared, to enlarge the UNI molecule. A known compound 1 (a racemic mixture) was synthesized by a slightly modified method of Funaki et al. ${ }^{19,29}$ and Hallahan et al. ${ }^{30}$ The azidation reaction ${ }^{30}$ of $\mathbf{1}$ gave the azide 2 to be used in a click reaction ${ }^{31}$ with commercially available acetylene compounds, which have linear alkyl chains including three alcohols and an acid, to give 11 kinds of UT (4A, 
$1 \mathrm{H}, 2 \mathrm{H}, 4 \mathrm{H}, 3,4,7,9,11,13$, and 15 ) as racemic compounds (Scheme 1$).{ }^{32}$ The click reactions were performed with sodium ascorbate and $\mathrm{CuSO}_{4}$ in THF. Although most click reactions between azide and acetylene derivatives quantitatively produce only one coupling product, the reactions using 2 gave at least three products, including UT, the main one. Although the structure of byproducts was not established, NMR analysis indicated that the conjugated system of 2 was involved in the side reaction. All of the racemic UT compounds were isolated using preparative-scale HPLC before conducting enzyme and biological assays.

Inhibitory activity against ABA 8'-hydroxylase (CYP707A) was examined using recombinant Arabidopsis CYP707A3 coexpressed with Arabidopsis P450 reductase (AR2) in E. coli. ${ }^{33}$ The activity was evaluated based on the decrease of the enzyme product, phaseic acid, caused by addition of a test compound at a concentration two times higher $(10 \mu \mathrm{M})$ than the substrate S-ABA $(5 \mu \mathrm{M}) .{ }^{34}$ The alkyl-type UT, except for the longest-chain UT15, exhibited high inhibitory activity (greater than $90 \%$ ), which was equivalent to that of S-UNI (Table 1). The mode of inhibition of UT4 was determined to be competitive by plotting the reaction velocities in the presence and absence of inhibitor on a double-reciprocal plot; the inhibition constant $\left(K_{\mathrm{I}}\right)$ was $195 \mathrm{nM}$. Although this is larger than the value for S-UNI (10 nM), UT4 has strong affinity to the active site of ABA 8'-hydroxylase, considering that the $K_{\mathrm{M}}$ value for (+)-ABA was 3.4 $\mu \mathrm{M}$ under the same experimental conditions. The alcohol-types UT1H, UT2H, and UT4H were also effective $(75-85 \%$ inhibition) but were less potent than the alkyl-types. The acid-type UT4A was much less effective than the alcohol-types. These results suggest the following: (1) linear elongation at $\mathrm{C} 4$ of the phenyl in UNI is generally not crucial to binding to the active site of ABA 8'-hydroxylase; (2) the protic group in the elongated portion of the molecule may have a negative effect on binding to the enzyme, and (3) there is an upper limit to the length and hydrophobicity of the elongated portion that allows binding to the enzyme.

The alkyl-types of UT are highly hydrophobic; UT7-15 had an especially high estimated log $P$ value $(>5)$ (Table 1). Compounds with very high $\log P$ value are too poorly water soluble. In 
fact, UT7-15 did not dissolve in water, although it dissolved in the buffer used in the enzyme assay. These compounds are not practical to use as a plant regulator although they may be useful as chemical probes for in vitro enzymatic studies. Thus, only the UT compounds with a $\log P$ value $<4$ were assayed for rice seedling arrest. ${ }^{35}$ The acid- and alcohol-types of UT were not effective even at $100 \mu \mathrm{M}$, whereas the alkyl-types showed a significant effect at a concentration greater than $30 \mu \mathrm{M}$ (Figure 2). These results suggest that elongation at $\mathrm{C} 4$ of the phenyl in UNI depresses the affinity to enzymes involved in seedling growth; the hydrophilic substitution is especially crucial.

Considering its potency in ABA 8'-hydroxylase inhibition, its small effect on seedling growth, and its ease of application, UT4 was the best candidate for a highly selective inhibitor of ABA 8'-hydroxylase. To test the effect of UT4 on drought tolerance, 90-day-old apple seedlings were sprayed with an aqueous solution containing UT4 at concentrations of 10,50 , and $100 \mu \mathrm{M}$ for cultivar Akitabeniakari (Figure 3) and $10 \mu \mathrm{M}$ for Fuji (Figure 4). ${ }^{36}$ The UT4-sprayed seedlings showed drought tolerance at all tested concentrations. Although UNI showed a similar effect, browning of Akitabeniakari leaves was observed at $100 \mu \mathrm{M}$. This side effect, which may be caused by inhibition of other enzymes including P450s, was never observed at $100 \mu \mathrm{M}$ UT4 treatment. This result suggests that UT4 functions as a more selective inhibitor of ABA 8'-hydroxylase than UNI. In this research, we used racemic UT compounds. In the case of UNI, the $S$-enantiomer are effective in plants, whereas the $R$-enantiomer are ineffective in plants but act as fungicides. ${ }^{37,38}$ Therefore, the activity of racemic UT compounds in present assays may have been caused by the $S$-enantiomers. We plan to prepare optically pure UT compounds to verify whether UT has the same trend observed for UNI.

\section{Acknowledgments}


We thank Toray Industries, Inc. (Tokyo, Japan) for the gift of (+)-ABA. This research was supported by a Grant-in-Aid for Scientific Research (No. 18380192) from the Ministry of Education, Culture, Sports, Science and Technology of Japan.

\section{References and notes}

1. Hirai, N. Abscisic acid. In Comprehensive Natural Products Chemistry Vol. 8; Mori, K.

Ed.; Elsevier: Amsterdam, 1999; pp 72-91.

2. Davies, W. J.; Jones, H. G. Abscisic Acid; BIOS Scientific Publishers: Oxford, 1991.

3. Zeevaart, J. A. D.; Creelman, R. A. Annu. Rev. Plant Physiol. Plant Mol. Biol. 1998, 39, 439.

4. Nambara, E.; Marion-Poll, A. Annu. Rev. Plant Biol. 2005, 56, 165.

5. Huang, D.; Jaradat, MR.; Wu, W.; Ambrose, S. J.; Ross, A. R.; Abrams, S. R.; Cutler, A. J. Plant J. 2007, 50, 414.

6. Loveys, B. R. In Abscisic Acid; Davies, W. J.; Jones, H. G. Eds; BIOS Scientific Publishers: Oxford, 1991.

7. Saito, S.; Hirai, N.; Matsumoto, C.; Ohigashi, H.; Ohta, D.; Sakata, K.; Mizutani, M. Plant Physiol. 2004, 134, 1439.

8. Kushiro, T.; Okamoto, M.; Nakabayashi, K.; Yamagishi, K.; Kitamura, S.; Asami, T.; Hirai, N.; Koshiba, T.; Kamiya, Y.; Nambara, E. ENBO J. 2004, 23, 1647.

9. Yang, S. H.; Choi, D. Biochem. Biophys. Res. Commun. 2006, 350, 685.

10. Yang, S. H.; Zeevaart, J. A. D. Plant J. 2006, 47, 675. 
11. Millar, A. A.; Jacobsen, J. V.; Ross, J. J.; Helliwell, C. A.; Poole, A. T.; Scofield, G.; Reid, J. B.; Gubler, F. Plant J. 2006, 45, 942.

12. Destefano-Beltran, L.; Knauber, D.; Huckle, L.; Suttle, J. C. Plant Mol Biol. 2006, 61, 687.

13. Umezawa, T.; Okamoto, M.; Kushiro, T.; Nambara, E.; Oono, Y.; Seki, M.; Kobayashi, M.; Koshiba, T.; Kamiya, Y.; Shinozaki, K. Plant J. 2006, 46, 171.

14. Okamoto, M.; Kuwahara, A.; Seo, M.; Kushiro, T.; Asami, T.; Hirai, N.; Kamiya, Y.; Koshiba, T.; Nambara, E. Plant Physiol. 2006, 141, 97.

15. Ueno, K.; Araki, Y.; Hirai, N.; Saito, S.; Mizutani, M.; Sakata, K.; Todoroki, Y. Bioorg. Med. Chem. 2005, 13, 3359.

16. Ueno, K.; Yoneyama, H.; Saito, S.; Mizutani, M.; Sakata, K.; Hirai, N.; Todoroki, Y. Bioorg. Med. Chem. Lett. 2005, 15, 5226.

17. Araki, Y.; Miyawaki, A.; Miyashita, T.; Mizutani, M.; Hirai, N.; Todoroki, Y. Bioorg. Med. Chem. Lett. 2006, 16, 3302.

18. Funaki, Y.; Ishiguri, T.; Kato, T.; Tanaka, S. J. Pesticide Sci. 1984, 9, 229.

19. Funaki, Y.; Oshita, H.; Yamamoto, S.; Tanaka, S.; Kato, T. (Sumitomo Chemical Co.). Ger. Offen. DE3010560, 1980.

20. Kitahata, N.; Saito, S.; Miyazawa, Y.; Umezawa, T.; Shimada, Y.; Min, Y. K.; Mizutani, M.; Hirai, N.; Shinozaki, K.; Yoshida, S.; Asami, T. Bioorg. Med. Chem. 2005, 13, 4491.

21. Saito, S.; Okamoto, M.; Shinoda, S.; Kushiro, T.; Koshiba, T.; Kamiya, Y.; Hirai, N.; Todoroki, Y.; Sakata, K.; Nambara, E.; Mizutani, M. Biosci. Biotechnol. Biochem. 2006, 70, 1731.

22. Todoroki, Y.; Kobayashi, K.; Yoneyama, H.; Hiramatsu, S.; Jin, M.-H.; Watanabe, B.; 
Mizutani, M., Hirai, N. Bioorg. Med. Chem. 2008, 16, 3141.

23. Izumi, K.; Kamiya, Y.; Sakurai, A.; Oshio, H.; Takahashi, N. Plant Cell Physiol. 1985, 26, 821.

24. Iwasaki, T.; Shibaoka, H. Plant Cell Physiol. 1991, 32, 1007.

25. Asami, T.; Mizutani, M.; Fujioka, S.; Goda, H.; Min, Y. K.; Shimada, Y.; Nakano, T.; Takatsuto, S.; Matsuyama, T.; Nagata, N.; Sakata, K.; Yoshida, S. J. Biol. Chem. 2001, 276, 25687.

26. Izumi, K.; Nakagawa, S.; Kobayashi, M.; Oshio, H.; Sakurai, A.; Takahashi, N. Plant Cell Physiol. 1988, 29, 97.

27. Gotoh, O. J. Biol. Chem. 1992, 267, 83.

28. Kolb, H. C.; Sharpless, K. B. Drug Discovery Today 2003, 8, 1128, and references cited therein.

29. Funaki, Y.; Ishiguri, Y.; Kato, T.; Tanaka, S. J. Pesticide Sci. 1984, 9, 229.

30. Hallahan, D. L.; Heasman, A. P.; Grossel, M. C.; Quigley, R.; Hedden, P.; Bowyer, J. R. Plant Physiol. 1988, 88, 1425. 2: ${ }^{1} \mathrm{H}$ NMR (270 MHz, acetone- $\left.d_{6}\right): \delta 0.69$ (9H, s, $t$-butyl), 4.77 $(1 \mathrm{H}, \mathrm{d}, J=6.3 \mathrm{~Hz}, \mathrm{H}-3), 5.10(1 \mathrm{H}, \mathrm{d}, J=6.3 \mathrm{~Hz}, \mathrm{HO}-3), 7.09(1 \mathrm{H}, \mathrm{s}, \mathrm{H}-1), 7.18$ and 7.52 (each 2H, m, 4-N 3 -phenyl), 8.02 (1H, s, H-3"), 8.82 (1H, s, H-5"); HRMS (FAB): calcd for $\mathrm{C}_{15} \mathrm{H}_{19} \mathrm{ON}_{6}[\mathrm{M}+\mathrm{H}]^{+}$299.1620, found 299.1628.

31. Kolb, H. C.; Finn, M. G.; Sharpless, K. B. Angew. Chem. Int. Ed. Engl. 2001, 40, 2004.

32. To a stirred solution of $2(20 \mathrm{mg}, 67 \mu \mathrm{mol})$ and alkyne $(67 \mu \mathrm{mol})$ in THF $(10 \mathrm{~mL})$ was added aqueous $\mathrm{CuSO}_{4}(100 \mathrm{mM}, 10 \mathrm{~mL})$ and aqueous sodium ascorbate $(100 \mathrm{mM}, 10 \mathrm{~mL})$ at room temperature. The mixture was stirred for $1 \mathrm{~h}$ before extraction with EtOAc $(30 \mathrm{~mL} \times 3)$. The organic layer was washed with brine, dried over $\mathrm{Na}_{2} \mathrm{SO}_{4}$, and concentrated in vacuo. After filtering through a $0.2-\mu \mathrm{M}$ acetyl cellulose membrane filter (YMC Duo-Filter QDUO 15), the 
filtrate was purified by semipreparative HPLC with a reverse phase C18 column (YMC ODS-AQ, $150 \times 20 \mathrm{~mm})$ to obtain UT (30-40\% yield). UT4A: ${ }^{1} \mathrm{H}$ NMR $(270 \mathrm{MHz}$, acetone- $\left.d_{6}\right): \delta 0.68\left(9 \mathrm{H}, \mathrm{s}, t\right.$-butyl), $2.19\left(2 \mathrm{H}, \mathrm{m},-\mathrm{CH}_{2}-\mathrm{C}_{2}-\mathrm{CH}_{2}-\mathrm{COOH}\right), 2.34(2 \mathrm{H}, \mathrm{m}$, $\left.-\mathrm{CH}_{2}-\mathrm{CH}_{2}-\underline{\mathrm{H}}_{2}-\mathrm{COOH}\right), 2.84\left(2 \mathrm{H}, \mathrm{t}, J=7.6 \mathrm{~Hz},-\underline{\mathrm{C}}_{2}-\mathrm{CH}_{2}-\mathrm{CH}_{2}-\mathrm{COOH}\right), 4.78(1 \mathrm{H}, \mathrm{s}, \mathrm{H}-3)$, $7.18(1 \mathrm{H}, \mathrm{s}, \mathrm{H}-1), 7.63$ and 7.93 (each $2 \mathrm{H}, \mathrm{m}$, phenyl), 8.10, 8.38, and 8.95 (each 1H, s, 1,2,4-triazole and 1,2,3-triazole); HRMS (ESI-TOF, positive mode): calcd for $\mathrm{C}_{21} \mathrm{H}_{26} \mathrm{~N}_{6} \mathrm{O}_{3} \mathrm{Na}$ $[\mathrm{M}+\mathrm{Na}]^{+}$433.1964, found 433.1962. UT1H: ${ }^{1} \mathrm{H}$ NMR $\left(270 \mathrm{MHz}\right.$, acetone- $\left.d_{6}\right): \delta 0.68(9 \mathrm{H}, \mathrm{s}$, t-butyl), 4.71 (1H, s, H-3), 7.19 (1H, s, H-1), 7.65 and 7.93 (each 2H, m, phenyl), 8.11, 8.51, and 8.96 (each 1H, s, 1,2,4-triazole and 1,2,3-triazole); HRMS (ESI-TOF, positive mode): calcd for $\mathrm{C}_{18} \mathrm{H}_{22} \mathrm{~N}_{6} \mathrm{O}_{2} \mathrm{Na}[\mathrm{M}+\mathrm{Na}]^{+}$377.1701, found 377.1705. UT2H: ${ }^{1} \mathrm{H}$ NMR $(270 \mathrm{MHz}$, acetone- $\left.d_{6}\right): \delta 0.68\left(9 \mathrm{H}, \mathrm{s}, t\right.$-butyl), $1.60\left(3 \mathrm{H}, \mathrm{d}, J=6.6 \mathrm{~Hz},-\mathrm{CH}(\mathrm{OH})-\underline{\mathrm{H}}_{3}\right), 4.71(1 \mathrm{H}, \mathrm{s}, \mathrm{H}-3)$, $5.06\left(1 \mathrm{H}, \mathrm{q}, J=6.6 \mathrm{~Hz},-\mathrm{C} \underline{\mathbf{H}}(\mathrm{OH})-\mathrm{CH}_{3}\right), 7.18(1 \mathrm{H}, \mathrm{s}, \mathrm{H}-1), 7.64$ and 7.94 (each 2H, m, phenyl), 8.11, 8.46, and 8.95 (each 1H, s, 1,2,4-triazole and 1,2,3-triazole); HRMS (ESI-TOF, positive mode): calcd for $\mathrm{C}_{19} \mathrm{H}_{24} \mathrm{~N}_{6} \mathrm{O}_{2} \mathrm{Na}[\mathrm{M}+\mathrm{Na}]^{+}$391.1858, found 391.1865. UT4H: ${ }^{1} \mathrm{H}$ NMR (270 $\mathrm{MHz}$, acetone- $\left.d_{6}\right): \delta 0.67\left(9 \mathrm{H}, \mathrm{s}, t\right.$-butyl), $0.93\left(3 \mathrm{H}, \mathrm{t}, J=7.6 \mathrm{~Hz},-\mathrm{CH}(\mathrm{OH})-\left(\mathrm{CH}_{2}\right)_{2}-\mathrm{C}_{3}\right), 1.47$ and 2.08 (each $\left.2 \mathrm{H}, \mathrm{m},-\mathrm{CH}(\mathrm{OH})-\left(\mathrm{CH}_{2}\right)_{2}-\mathrm{CH}_{3}\right), 4.78(1 \mathrm{H}, \mathrm{s}, \mathrm{H}-3), 4.89(1 \mathrm{H}, \mathrm{t}, J=6.6 \mathrm{~Hz}$, $\left.-\mathrm{C} \underline{\mathbf{H}}(\mathrm{OH})-\left(\mathrm{CH}_{2}\right)_{2}-\mathrm{CH}_{3}\right), 7.16(1 \mathrm{H}, \mathrm{s}, \mathrm{H}-1), 7.67$ and 7.97 (each $2 \mathrm{H}, \mathrm{m}$, phenyl), 8.02, 8.43, and 8.87 (each 1H, s, 1,2,4-triazole and 1,2,3-triazole); HRMS (ESI-TOF, positive mode): calcd for $\mathrm{C}_{21} \mathrm{H}_{28} \mathrm{~N}_{6} \mathrm{O}_{2} \mathrm{Na}[\mathrm{M}+\mathrm{Na}]^{+}$419.2171, found 419.2175. UT3: ${ }^{1} \mathrm{H}$ NMR $\left(270 \mathrm{MHz}, \mathrm{CD}_{3} \mathrm{OD}\right): \delta$ $0.68\left(9 \mathrm{H}, \mathrm{s}, t\right.$-butyl), $1.02\left(3 \mathrm{H}, \mathrm{t}, J=7.6 \mathrm{~Hz},-\mathrm{CH}_{2}-\mathrm{CH}_{2}-\mathrm{C}_{-}\right), 1.78(2 \mathrm{H}, \mathrm{tq}, J=7.6$ and $7.6 \mathrm{~Hz}$, $\left.-\mathrm{CH}_{2}-\underline{\mathrm{C}}_{2}-\mathrm{CH}_{3}\right), 2.77\left(2 \mathrm{H}, \mathrm{t}, J=7.6 \mathrm{~Hz},-\underline{\mathrm{C}}_{2}-\mathrm{CH}_{2}-\mathrm{CH}_{3}\right), 4.71(1 \mathrm{H}, \mathrm{s}, \mathrm{H}-3), 7.17(1 \mathrm{H}, \mathrm{s}, \mathrm{H}-1)$, 7.64 and 7.92 (each 2H, m, phenyl), 8.11, 8.35, and 8.95 (each 1H, s, 1,2,4-triazole and 1,2,3-triazole); HRMS (ESI-TOF, positive mode): calcd for $\mathrm{C}_{20} \mathrm{H}_{26} \mathrm{~N}_{6} \mathrm{ONa}[\mathrm{M}+\mathrm{Na}]^{+} 389.2065$, found 389.2065. UT4: ${ }^{1} \mathrm{H}$ NMR (270 MHz, $\left.\mathrm{CD}_{3} \mathrm{OD}\right): \delta 0.68(9 \mathrm{H}, \mathrm{s}, t$-butyl), $0.98(3 \mathrm{H}, \mathrm{t}, J=$ $\left.7.6 \mathrm{~Hz},-\mathrm{CH}_{2}-\mathrm{CH}_{2}-\mathrm{CH}_{2}-\underline{\mathrm{H}}_{3}\right), 1.44\left(2 \mathrm{H}, \mathrm{tq}, J=7.6\right.$ and $\left.7.6 \mathrm{~Hz},-\mathrm{CH}_{2}-\mathrm{CH}_{2}-\underline{\mathrm{C}}_{2}-\mathrm{CH}_{3}\right), 1.74(2 \mathrm{H}$, tt, $J=7.6$ and $\left.7.6 \mathrm{~Hz},-\mathrm{CH}_{2}-\underline{\mathrm{C}}_{2}-\mathrm{CH}_{2}-\mathrm{CH}_{3}\right), 2.79\left(2 \mathrm{H}, \mathrm{t}, J=7.6 \mathrm{~Hz},-\underline{\mathrm{C}}_{2}-\mathrm{CH}_{2}-\mathrm{CH}_{2}-\mathrm{CH}_{3}\right), 4.71$ (1H, s, H-3), 7.17 (1H, s, H-1), 7.63 and 7.92 (each 2H, m, phenyl), 8.11, 8.34, and 8.95 (each 1H, s, 1,2,4-triazole and 1,2,3-triazole); HRMS (ESI-TOF, positive mode): calcd for 
$\mathrm{C}_{21} \mathrm{H}_{28} \mathrm{~N}_{6} \mathrm{ONa}[\mathrm{M}+\mathrm{Na}]^{+}$403.2281, found 403.2273. UT7: ${ }^{1} \mathrm{H} \mathrm{NMR}\left(270 \mathrm{MHz}, \mathrm{CD}_{3} \mathrm{OD}\right): \delta$ $0.68\left(9 \mathrm{H}, \mathrm{s}, \mathrm{t}\right.$-butyl), $0.88\left(3 \mathrm{H}, \mathrm{t}, J=6.6 \mathrm{~Hz},-\mathrm{CH}_{2}-\mathrm{CH}_{2}-\left(\mathrm{CH}_{2}\right)_{4}-\mathrm{C}_{3}\right), 1.32(8 \mathrm{H}, \mathrm{m}$, $\left.-\mathrm{CH}_{2}-\mathrm{CH}_{2}-\left(\mathrm{C}_{2}\right)_{4}-\mathrm{CH}_{3}\right), 1.76\left(2 \mathrm{H}, \mathrm{m},-\mathrm{CH}_{2}-\underline{\mathrm{C}}_{2}-\left(\mathrm{CH}_{2}\right)_{4}-\mathrm{CH}_{3}\right), 2.79(2 \mathrm{H}, \mathrm{t}, J=7.3 \mathrm{~Hz}$, $\left.-\underline{\mathrm{H}}_{2}-\mathrm{CH}_{2}-\left(\mathrm{CH}_{2}\right)_{6}-\mathrm{CH}_{3}\right), 4.71(1 \mathrm{H}, \mathrm{s}, \mathrm{H}-3), 7.17(1 \mathrm{H}, \mathrm{s}, \mathrm{H}-1), 7.63$ and 7.92 (each $2 \mathrm{H}, \mathrm{m}$, phenyl), 8.11, 8.34, and 8.95 (each 1H, s, 1,2,4-triazole and 1,2,3-triazole); HRMS (ESI-TOF, positive mode): calcd for $\mathrm{C}_{24} \mathrm{H}_{34} \mathrm{~N}_{6} \mathrm{ONa}[\mathrm{M}+\mathrm{Na}]^{+}$445.2692, found 445.2690. UT9: ${ }^{1} \mathrm{H}$ NMR (270 MHz, CD 3 OD): $\delta 0.68$ (9H, s, t-butyl), $0.88\left(3 \mathrm{H}, \mathrm{t}, J=6.6 \mathrm{~Hz},-\mathrm{CH}_{2}-\mathrm{CH}_{2}-\left(\mathrm{CH}_{2}\right)_{6}-\mathrm{C}_{3}\right)$, 1.30-1.38 (12H, m, $\left.-\mathrm{CH}_{2}-\mathrm{CH}_{2}-\left(\mathrm{C}_{2}\right)_{6}-\mathrm{CH}_{3}\right), 1.74(2 \mathrm{H}, \quad \mathrm{tt}, \quad J=7.6$ and $7.6 \mathrm{~Hz}$, $\left.-\mathrm{CH}_{2}-\underline{\mathrm{C}}_{2}-\left(\mathrm{CH}_{2}\right)_{6}-\mathrm{CH}_{3}\right), 2.78\left(2 \mathrm{H}, \mathrm{t}, J=7.6 \mathrm{~Hz},-\underline{\mathrm{H}}_{2}-\mathrm{CH}_{2}-\left(\mathrm{CH}_{2}\right)_{6}-\mathrm{CH}_{3}\right), 4.78(1 \mathrm{H}, \mathrm{s}, \mathrm{H}-3)$, $7.17(1 \mathrm{H}, \mathrm{s}, \mathrm{H}-1), 7.63$ and 7.92 (each $2 \mathrm{H}, \mathrm{m}$, phenyl), 8.11, 8.34, and 8.95 (each 1H, s, 1,2,4-triazole and 1,2,3-triazole); HRMS (ESI-TOF, positive mode): calcd for $\mathrm{C}_{26} \mathrm{H}_{38} \mathrm{~N}_{6} \mathrm{ONa}$ $[\mathrm{M}+\mathrm{Na}]^{+}$473.3004, found 473.3003. UT10: ${ }^{1} \mathrm{H} \mathrm{NMR}\left(270 \mathrm{MHz}, \mathrm{CD}_{3} \mathrm{OD}\right): \delta 0.68(9 \mathrm{H}, \mathrm{s}$, t-butyl), $\quad 0.89\left(3 \mathrm{H}, \quad \mathrm{t}, \quad J=6.9 \mathrm{~Hz}, \quad-\mathrm{CH}_{2}-\mathrm{CH}_{2}-\left(\mathrm{CH}_{2}\right)_{7}-\underline{\mathrm{C}}_{3}\right), \quad 1.29 \quad(14 \mathrm{H}, \mathrm{m}$, $\left.-\mathrm{CH}_{2}-\mathrm{CH}_{2}-\left(\mathrm{C}_{2}\right)_{7}-\mathrm{CH}_{3}\right), 1.76\left(2 \mathrm{H}, \mathrm{tt}, J=7.6\right.$ and $\left.7.6 \mathrm{~Hz},-\mathrm{CH}_{2}-\mathrm{C}_{2}-\left(\mathrm{CH}_{2}\right)_{7}-\mathrm{CH}_{3}\right), 2.79(2 \mathrm{H}, \mathrm{t}, J$ $\left.=7.6 \mathrm{~Hz},-\underline{\mathrm{H}}_{2}-\mathrm{CH}_{2}-\left(\mathrm{CH}_{2}\right)_{7}-\mathrm{CH}_{3}\right), 4.71(1 \mathrm{H}, \mathrm{s}, \mathrm{H}-3), 7.17(1 \mathrm{H}, \mathrm{s}, \mathrm{H}-1), 7.63$ and $7.92($ each $2 \mathrm{H}$, $\mathrm{m}$, phenyl), 8.10, 8.34, and 8.95 (each 1H, s, 1,2,4-triazole and 1,2,3-triazole); HRMS (ESI-TOF, positive mode): calcd for $\mathrm{C}_{27} \mathrm{H}_{40} \mathrm{~N}_{6} \mathrm{ONa}[\mathrm{M}+\mathrm{Na}]^{+}$487.3161, found 487.3167. UT11: ${ }^{1} \mathrm{H}$ NMR $\left(270 \mathrm{MHz}, \mathrm{CD}_{3} \mathrm{OD}\right): \delta 0.68(9 \mathrm{H}, \mathrm{s}, t$-butyl), $0.89(3 \mathrm{H}, \mathrm{t}, J=6.9 \mathrm{~Hz}$, $\left.-\mathrm{CH}_{2}-\mathrm{CH}_{2}-\left(\mathrm{CH}_{2}\right)_{8}-\underline{\mathrm{C}}_{3}\right), 1.29\left(16 \mathrm{H}, \mathrm{m},-\mathrm{CH}_{2}-\mathrm{CH}_{2}-\left(\mathrm{C}_{2}\right)_{8}-\mathrm{CH}_{3}\right), 1.76(2 \mathrm{H}, \mathrm{tt}, J=7.6$ and 7.6 $\left.\mathrm{Hz},-\mathrm{CH}_{2}-\underline{\mathrm{C}}_{2}-\left(\mathrm{CH}_{2}\right)_{8}-\mathrm{CH}_{3}\right), 2.79\left(2 \mathrm{H}, \mathrm{t}, J=7.6 \mathrm{~Hz},-\underline{\mathrm{C}}_{2}-\mathrm{CH}_{2}-\left(\mathrm{CH}_{2}\right)_{8}-\mathrm{CH}_{3}\right), 4.71(1 \mathrm{H}, \mathrm{s}, \mathrm{H}-3)$, $7.18(1 \mathrm{H}, \mathrm{s}, \mathrm{H}-1), 7.63$ and 7.92 (each $2 \mathrm{H}, \mathrm{m}$, phenyl), 8.11, 8.35, and 8.95 (each $1 \mathrm{H}, \mathrm{s}$, 1,2,4-triazole and 1,2,3-triazole); HRMS (ESI-TOF, positive mode): calcd for $\mathrm{C}_{28} \mathrm{H}_{42} \mathrm{~N}_{6} \mathrm{ONa}$ $[\mathrm{M}+\mathrm{Na}]^{+}$501.3317, found 501.3318. UT12: ${ }^{1} \mathrm{H} \mathrm{NMR}\left(270 \mathrm{MHz}, \mathrm{CD}_{3} \mathrm{OD}\right): \delta 0.68(9 \mathrm{H}, \mathrm{s}$, $t$-butyl), $\quad 0.87\left(3 \mathrm{H}, \quad \mathrm{t}, \quad J=6.9 \mathrm{~Hz}, \quad-\mathrm{CH}_{2}-\mathrm{CH}_{2}-\left(\mathrm{CH}_{2}\right)_{9}-\underline{C}_{3}\right), \quad 1.33 \quad(18 \mathrm{H}, \quad \mathrm{m}$, $\left.-\mathrm{CH}_{2}-\mathrm{CH}_{2}-\left(\mathrm{C}_{2}\right)_{9}-\mathrm{CH}_{3}\right), 1.75\left(2 \mathrm{H}, \mathrm{tt}, J=7.6\right.$ and $\left.7.6 \mathrm{~Hz},-\mathrm{CH}_{2}-\mathrm{C}_{2}-\left(\mathrm{CH}_{2}\right)_{9}-\mathrm{CH}_{3}\right), 2.78(2 \mathrm{H}, \mathrm{t}, J$ $\left.=7.6 \mathrm{~Hz},-\underline{\mathrm{H}}_{2}-\mathrm{CH}_{2}-\left(\mathrm{CH}_{2}\right)_{9}-\mathrm{CH}_{3}\right), 4.71(1 \mathrm{H}, \mathrm{s}, \mathrm{H}-3), 7.17(1 \mathrm{H}, \mathrm{s}, \mathrm{H}-1), 7.63$ and $7.92($ each $2 \mathrm{H}$, $\mathrm{m}$, phenyl), 8.11, 8.35, and 8.95 (each 1H, s, 1,2,4-triazole and 1,2,3-triazole); HRMS (ESI-TOF, positive mode): calcd for $\mathrm{C}_{29} \mathrm{H}_{44} \mathrm{~N}_{6} \mathrm{ONa}[\mathrm{M}+\mathrm{Na}]^{+}$515.3474, found 515.3480. 
UT13: ${ }^{1} \mathrm{H}$ NMR $\left(270 \mathrm{MHz}, \mathrm{CD}_{3} \mathrm{OD}\right): \delta 0.68(9 \mathrm{H}, \mathrm{s}, t$-butyl), $0.89(3 \mathrm{H}, \mathrm{t}, J=6.9 \mathrm{~Hz}$, $\left.-\mathrm{CH}_{2}-\mathrm{CH}_{2}-\left(\mathrm{CH}_{2}\right)_{10}-\mathrm{C}_{3}\right), 1.33\left(20 \mathrm{H}, \mathrm{m},-\mathrm{CH}_{2}-\mathrm{CH}_{2}-\left(\mathrm{C}_{2}\right)_{10}-\mathrm{CH}_{3}\right), 1.75(2 \mathrm{H}, \mathrm{tt}, J=7.6$ and 7.6 $\left.\mathrm{Hz},-\mathrm{CH}_{2}-\underline{\mathrm{C}}_{2}-\left(\mathrm{CH}_{2}\right)_{10}-\mathrm{CH}_{3}\right), 2.79\left(2 \mathrm{H}, \mathrm{t}, J=7.6 \mathrm{~Hz},-\underline{\mathrm{C}}_{2}-\mathrm{CH}_{2}-\left(\mathrm{CH}_{2}\right)_{10}-\mathrm{CH}_{3}\right), 4.71(1 \mathrm{H}, \mathrm{s}$, H-3), 7.17 (1H, s, H-1), 7.63 and 7.92 (each 2H, m, phenyl), 8.11, 8.34, and 8.95 (each 1H, s, 1,2,4-triazole and 1,2,3-triazole); HRMS (ESI-TOF, positive mode): calcd for $\mathrm{C}_{30} \mathrm{H}_{46} \mathrm{~N}_{6} \mathrm{ONa}$ $[\mathrm{M}+\mathrm{Na}]^{+}$529.3630, found 529.3629. UT15: ${ }^{1} \mathrm{H}$ NMR $\left(270 \mathrm{MHz}, \mathrm{CD}_{3} \mathrm{OD}\right): \delta 0.68(9 \mathrm{H}, \mathrm{s}$, t-butyl), $\quad 0.88\left(3 \mathrm{H}, \quad \mathrm{t}, \quad J=7.2 \mathrm{~Hz}, \quad-\mathrm{CH}_{2}-\mathrm{CH}_{2}-\left(\mathrm{CH}_{2}\right)_{12}-\underline{\mathrm{C}}_{3}\right), \quad 1.33 \quad(24 \mathrm{H}, \quad \mathrm{m}$, $\left.-\mathrm{CH}_{2}-\mathrm{CH}_{2}-\left(\underline{\mathrm{C}}_{2}\right)_{12}-\mathrm{CH}_{3}\right), 1.73\left(2 \mathrm{H}, \mathrm{tt}, J=7.6\right.$ and $\left.7.6 \mathrm{~Hz},-\mathrm{CH}_{2}-\underline{\mathrm{C}}_{2}-\left(\mathrm{CH}_{2}\right)_{12}-\mathrm{CH}_{3}\right), 2.78(2 \mathrm{H}, \mathrm{t}$, $\left.J=7.6 \mathrm{~Hz},-\underline{\mathrm{C}}_{2}-\mathrm{CH}_{2}-\left(\mathrm{CH}_{2}\right)_{12}-\mathrm{CH}_{3}\right), 4.71(1 \mathrm{H}, \mathrm{s}, \mathrm{H}-3), 7.17(1 \mathrm{H}, \mathrm{s}, \mathrm{H}-1), 7.63$ and 7.92 (each $2 \mathrm{H}, \mathrm{m}$, phenyl), 8.11, 8.35, and 8.95 (each 1H, s, 1,2,4-triazole and 1,2,3-triazole); HRMS (ESI-TOF, positive mode): calcd for $\mathrm{C}_{32} \mathrm{H}_{50} \mathrm{~N}_{6} \mathrm{ONa}[\mathrm{M}+\mathrm{Na}]^{+}$557.3943, found 557.3953.

33. A truncated Arabidopsis CYP707A3 (707A3d28), which lacked the putative membrane-spanning segment of the N-terminus (residues 3-28) was constructed. Cells of $E$. coli strain BL21 were transformed with pCW-CYP707A3d28 and pACYC-AR2 constructs. Cultures $(3 \mathrm{~mL})$ were grown overnight in Luria-Bertani medium supplemented with ampicillin (50 $\left.\mu \mathrm{g} \mathrm{mL}^{-1}\right)$ and chloramphenicol $\left(100 \mu \mathrm{g} \mathrm{mL}^{-1}\right)$. Then, $50 \mathrm{~mL}$ of Terrific Broth medium supplemented with ampicillin $\left(50 \mu \mathrm{g} \mathrm{mL}^{-1}\right)$, chloramphenicol $\left(100 \mu \mathrm{g} \mathrm{mL}^{-1}\right)$, and aminolevulic acid $(0.5 \mathrm{mM})$ was inoculated with a $0.5-\mathrm{mL}$ aliquot of the overnight culture. The culture was incubated at $37^{\circ} \mathrm{C}$ with gentle shaking $\left(225 \mathrm{rpm}\right.$ ) until the $\mathrm{A}_{600}$ reached 0.6 (after 2.5-3 h), and then expression of the P450 enzyme was induced by the addition of isopropyl- $\beta$-D-thiogalactopyranoside $(0.1 \mathrm{mM})$. The culture was shaken continuously (150 $\mathrm{rpm}$ ) at $25^{\circ} \mathrm{C}$ and cells were harvested $48 \mathrm{~h}$ later by centrifugation at $2,330 \mathrm{~g}$ for $20 \mathrm{~min}$ at $4{ }^{\circ} \mathrm{C}$. Pelleted cells were suspended in $2.5 \mathrm{~mL}$ of a $50 \mathrm{mM}$ potassium phosphate buffer $(\mathrm{pH} 7.25)$ containing 20\% glycerol, $1 \mathrm{mM}$ EDTA, and $0.1 \mathrm{mM}$ dithiothreitol. The suspension was sonicated for $30 \mathrm{~s}$, centrifuged at $23,470 \mathrm{~g}$ for $30 \mathrm{~min}$ at $4{ }^{\circ} \mathrm{C}$, and the supernatant (enzyme solution) was collected. The P450 content was determined by spectrophotometric analysis using the extinction coefficient of a reduced CO difference spectrum $\left(91.1 \mathrm{mM}^{-1} \mathrm{~cm}^{-1}\right)$. 
34. Kinetic analysis was performed according to detailed protocols described previously. ${ }^{15} \mathrm{~A}$ reaction mixture containing $25 \mu \mathrm{g} \mathrm{mL}{ }^{-1}$ CYP707A3 microsomes coexpressed with AR2 in E. coli, (+)-ABA (final conc.: $0.5-128 \mu \mathrm{M}$ ), inhibitors $(2-1000 \mathrm{nM}$ in $5 \mu \mathrm{L} \mathrm{DMF}$ ) and $50 \mu \mathrm{M}$ NADPH in $50 \mathrm{mM}$ potassium phosphate buffer ( $\mathrm{pH} 7.25)$ were incubated for $10 \mathrm{~min}$ at $30^{\circ} \mathrm{C}$; controls contained no inhibitor. Reactions were initiated by adding NADPH, and stopped by addition of $50 \mu \mathrm{L}$ of $1 \mathrm{M} \mathrm{NaOH}$. The reaction mixtures were acidified with $100 \mu \mathrm{L}$ of $1 \mathrm{M} \mathrm{HCl}$. To extract the reaction products, the mixtures were loaded onto Oasis HLB cartridges $(1 \mathrm{~mL}, 30$ $\mathrm{mg}$; Waters) and washed with $1 \mathrm{~mL}$ of $10 \% \mathrm{MeOH}$ in a solution containing $0.5 \% \mathrm{AcOH}$. The enzyme products were then eluted with $1 \mathrm{~mL}$ of $\mathrm{MeOH}$ containing $0.5 \% \mathrm{AcOH}$, and the eluate was concentrated in vacuo. The dried sample was dissolved in $50 \mu \mathrm{L}$ of $\mathrm{MeOH}$, and $10 \mu \mathrm{L}$ was subjected to HPLC. HPLC conditions were: ODS column, Hydrosphere C18 $(150 \times 6.0 \mathrm{~mm}$, YMC); solvent, $35 \% \mathrm{MeOH}$ or $20 \% \mathrm{MeCN}$ in $\mathrm{H}_{2} \mathrm{O}$ containing $0.1 \% \mathrm{AcOH}$; flow rate, $1.0 \mathrm{~mL}$ $\min ^{-1}$; detection, $254 \mathrm{~nm}$. Enzyme activity was confirmed by determining the amounts of phaseic acid in control experiments before each set of measurements. Inhibition constants were determined using the Enzyme Kinetics module of SigmaPlot 10 software (2006; Systat Software, Inc.) after determining the mode of inhibition by plotting the reaction velocities in the presence and absence of inhibitor on a double-reciprocal plot. For the uninhibited enzymatic reaction, the $K_{\mathrm{M}}$ for $(+)$-ABA was calculated to be $3.4 \pm 0.6 \mu \mathrm{M}$ based on five separate experiments. All tests were conducted at least three times.

35. Seeds of rice (Oryza sativa L. cv. Nipponbare) were sterilized with EtOH for 5 min and washed with running tap water. The sterilized seeds were soaked in water and allowed to germinate for 3 days at $25{ }^{\circ} \mathrm{C}$. The seeds were then placed in a glass tube (40 mm i.d.) containing $2 \mathrm{~mL}$ of a test solution and grown with the tube sealed with a plastic cap under continuous light (10000 lux) at $25^{\circ} \mathrm{C}$. When the seedlings were 7 days old, the length of the second leaf sheath was measured, and the inhibition ratio was calculated. The inhibition ratio was defined as $[(A-B) / A] \times 100$, where $A=$ the mean length of the second leaf sheath when water was used, and $B=$ the mean length of the second leaf sheath when a test compound was used. All tests were conducted at least twice. 
36. Cultivar Akitabeniakari apple [Malus sylvestris L. Mill. var. domestica (Borkh.) Mansf.] seeds were soaked overnight in water, then sown in moist vermiculite and grown in a greenhouse. Seedlings at 90 days old were divided into two groups: water-stressed and well-watered. The seedlings of the water-stressed group were sprayed uniformly with $2 \mathrm{~mL}$ of a test solution $(10,50$, and $100 \mu \mathrm{M})$ containing UT4, UNI (positive control), or distilled water (untreated control) before water-stressing them for $150 \mathrm{~h}$, then watering them once, and then water-stressing them again for an additional $330 \mathrm{~h}$. The well-watered group was watered once daily so that the vermiculite was saturated. Fuji apple [Malus sylvestris (L.) Mill. var. domestica (Borkh.) Mansf.] seeds were soaked overnight in water, then sown in moist vermiculite and grown in a greenhouse. The seedlings were 60 days old at the time of treatment. Each plant was sprayed uniformly with $2 \mathrm{~mL}$ of a test solution. The untreated control group was sprayed with distilled water, and the treated group was sprayed with a solution of UT $(10 \mu \mathrm{M})$ or $S$-UNI (10 $\mu \mathrm{M})$. All test groups were watered before being treated. The seedlings were water-stressed for $480 \mathrm{~h}$.

37. Tanaka, S. Kagaku to Seibutsu 1986, 24, 180.

38. Takano, H.; Oguri, Y.; Kato, T. J. Pesticide Sci. 1986, 11, 373. 
Table, Figure, and Scheme legends

Table 1. Inhibitory activity of UT against recombinant CYP707A3 and rice seedling elongation.

Figure 1. $S$-UNI is a potent inhibitor of ABA 8'-hydroxylase and ent-kaurene oxidase.

Figure 2. Inhibitory effect of UT and S-UNI on rice seedling growth.

Figure 3. Drought tolerance of Akitabeniakari apple seedlings treated with (a) UT4 and (b) $S$-UNI.

Figure 4. Drought tolerance of Fuji apple seedlings treated with distilled water (left), $10 \mu \mathrm{M}$ of UT (center), and $10 \mu \mathrm{M}$ of $S$-UNI (right).

Scheme 1. Synthesis of UT. R: see Table 1. Reagents and conditions: (i) $\mathrm{HCl}, \mathrm{NaNO}_{2}$, urea, $\mathrm{NaN}_{3}, 0{ }^{\circ} \mathrm{C}, 75 \%$; (ii) alkyne, THF, $\mathrm{CuSO}_{4}$, sodium ascorbate, rt, 30-40\%. 
Table 1. Inhibitory activity of UT against recombinant CYP707A3 and rice seedling elongation.

\begin{tabular}{|c|c|c|c|c|}
\hline \multirow{2}{*}{ Compound } & \multicolumn{2}{|c|}{ Structural properties } & \multirow{2}{*}{$\begin{array}{c}\text { CYP707A3 } \\
\text { Inhibition }^{\mathrm{b}}(\%)\end{array}$} & \multirow{2}{*}{$\begin{array}{c}\text { Rice } \\
\mathrm{IC}_{50}{ }^{\mathrm{c}}(\mu \mathrm{M})\end{array}$} \\
\hline & $-\mathrm{R}$ & $\log P^{\mathrm{a}}$ & & \\
\hline$S$-UNI & - & 3.13 & $100\left(K_{\mathrm{I}}=10 \mathrm{nM}\right)$ & 0.18 \\
\hline UT4A & $-\left(\mathrm{CH}_{2}\right)_{3}-\mathrm{COOH}$ & 2.64 & 63 & $\mathrm{NI}^{\mathrm{d}}$ \\
\hline UT1H & $-\mathrm{CH}_{2} \mathrm{OH}$ & 1.55 & 83 & NI \\
\hline UT2H & $-\mathrm{CH}(\mathrm{OH})-\mathrm{CH}_{3}$ & 2.12 & 77 & NI \\
\hline UT4H & $-\mathrm{CH}(\mathrm{OH})-\left(\mathrm{CH}_{2}\right)_{2}-\mathrm{CH}_{3}$ & 3.08 & 92 & NI \\
\hline UT3 & $-\left(\mathrm{CH}_{2}\right)_{2}-\mathrm{CH}_{3}$ & 3.51 & 92 & 21 \\
\hline UT4 & $-\left(\mathrm{CH}_{2}\right)_{3}-\mathrm{CH}_{3}$ & 3.96 & $100\left(K_{\mathrm{I}}=195 \mathrm{nM}\right)$ & 42 \\
\hline UT7 & $-\left(\mathrm{CH}_{2}\right)_{6}-\mathrm{CH}_{3}$ & 5.29 & 96 & $\mathrm{NT}^{\mathrm{e}}$ \\
\hline UT9 & $-\left(\mathrm{CH}_{2}\right)_{8}-\mathrm{CH}_{3}$ & 6.18 & 100 & NT \\
\hline UT11 & $-\left(\mathrm{CH}_{2}\right)_{10}-\mathrm{CH}_{3}$ & 7.07 & 100 & NT \\
\hline UT13 & $-\left(\mathrm{CH}_{2}\right)_{12}-\mathrm{CH}_{3}$ & 7.96 & 95 & NT \\
\hline UT15 & $-\left(\mathrm{CH}_{2}\right)_{14}-\mathrm{CH}_{3}$ & 8.85 & 54 & NT \\
\hline
\end{tabular}

${ }^{\text {a }}$ Calculated partition coefficient (Marvin, ChemAxon, http://www.chemaxon.com/).

${ }^{\mathrm{b}}$ Inhibition ratio of compounds $(10 \mu \mathrm{M})$ in the 8 '-hydroxylation of $\mathrm{ABA}(5 \mu \mathrm{M})$ (average of two sets of experiments).

${ }^{\mathrm{c}}$ The concentration for $50 \%$ inhibition (average of at least two sets of experiments).

${ }^{\mathrm{d}}$ No significant inhibition even at $100 \mu \mathrm{M}$ (max concentration tested).

${ }^{\mathrm{e}}$ Not tested because the compound was not dissolved in water. 


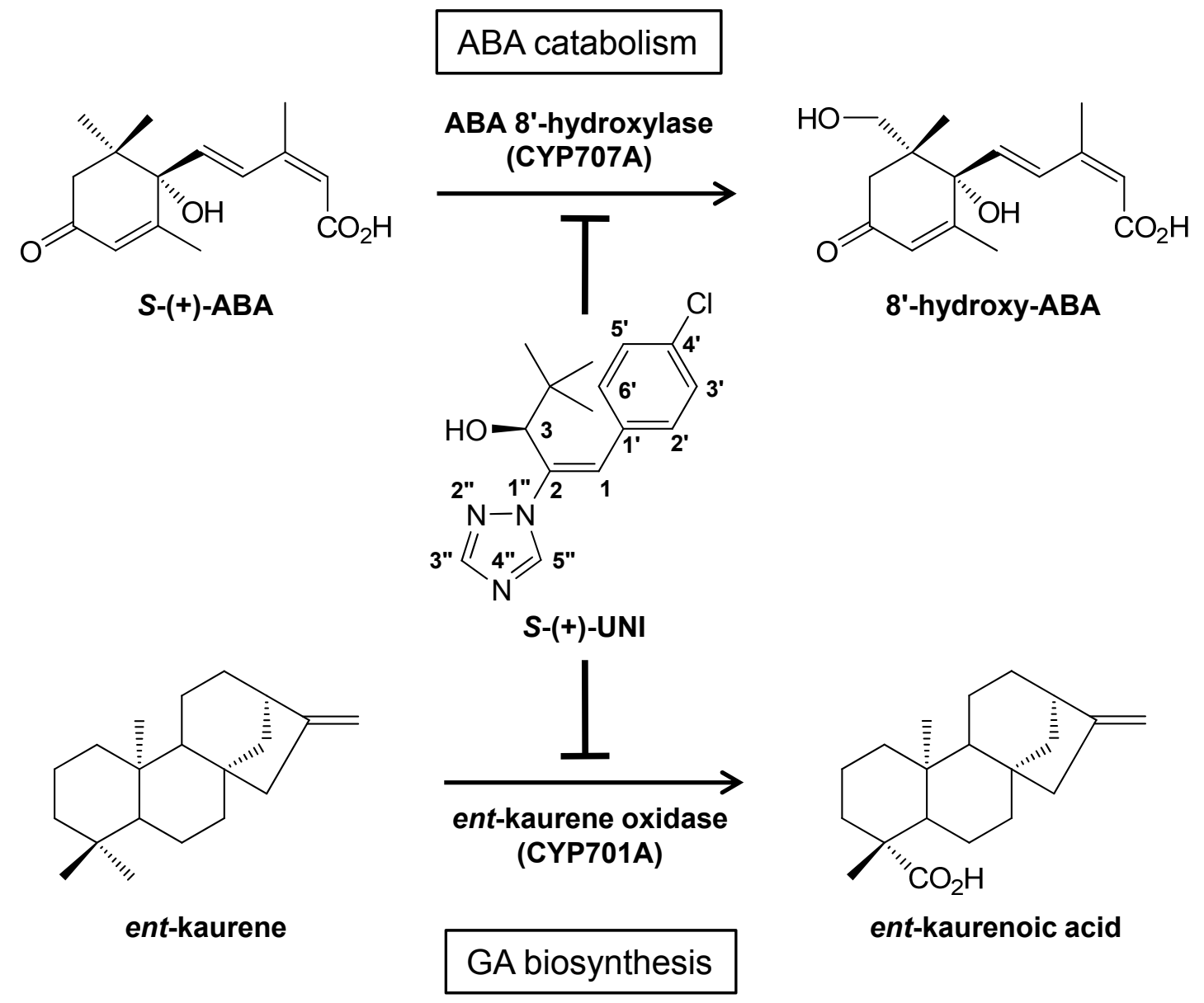

Figure 1 


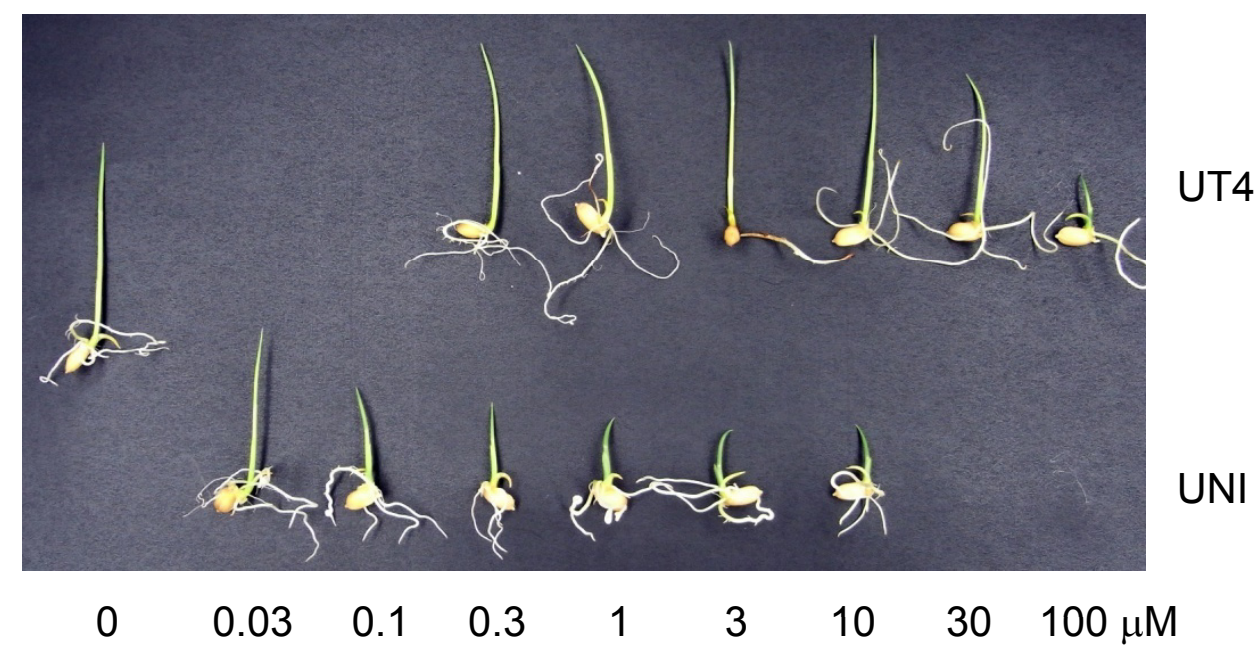

Figure 2 

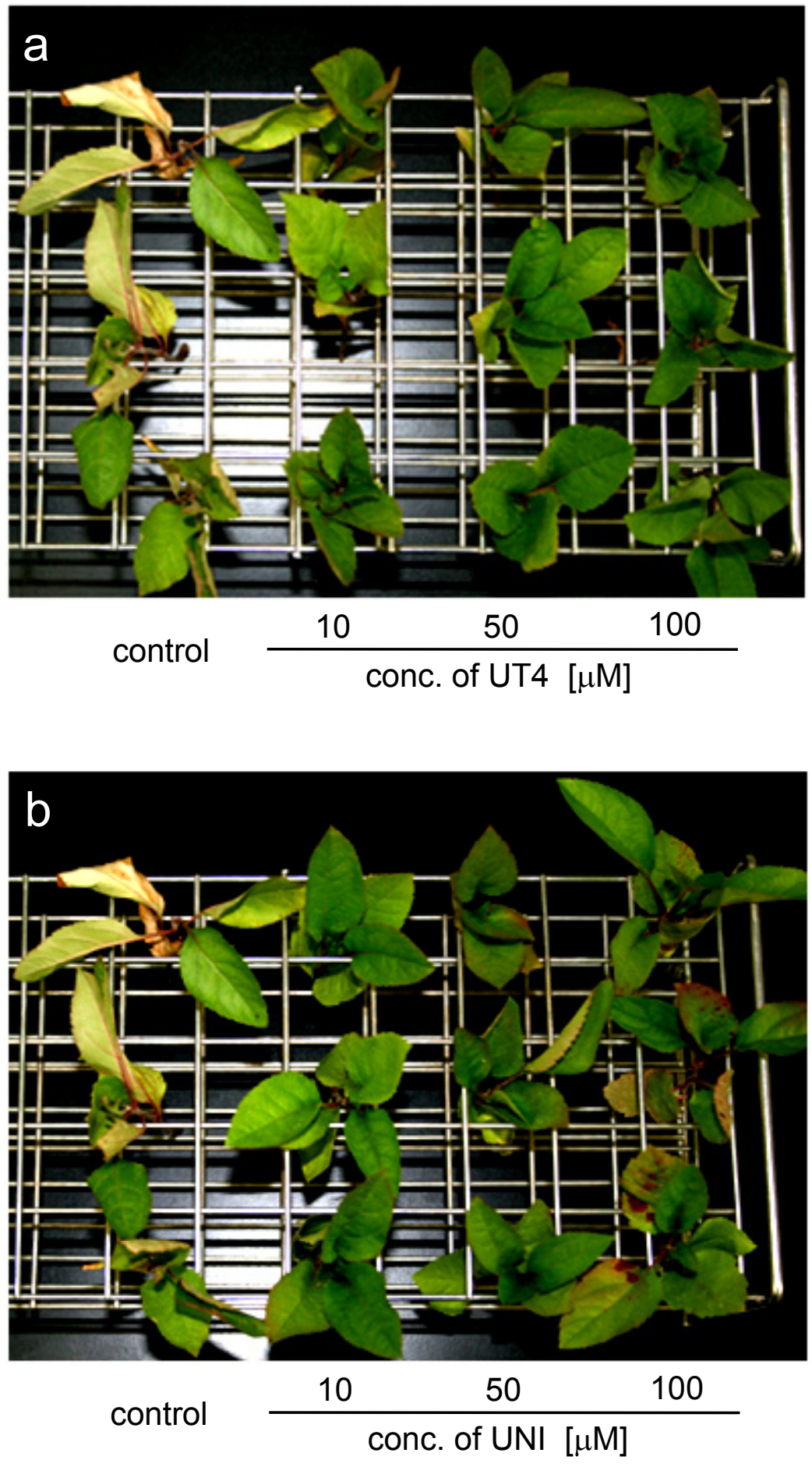

Figure 3 


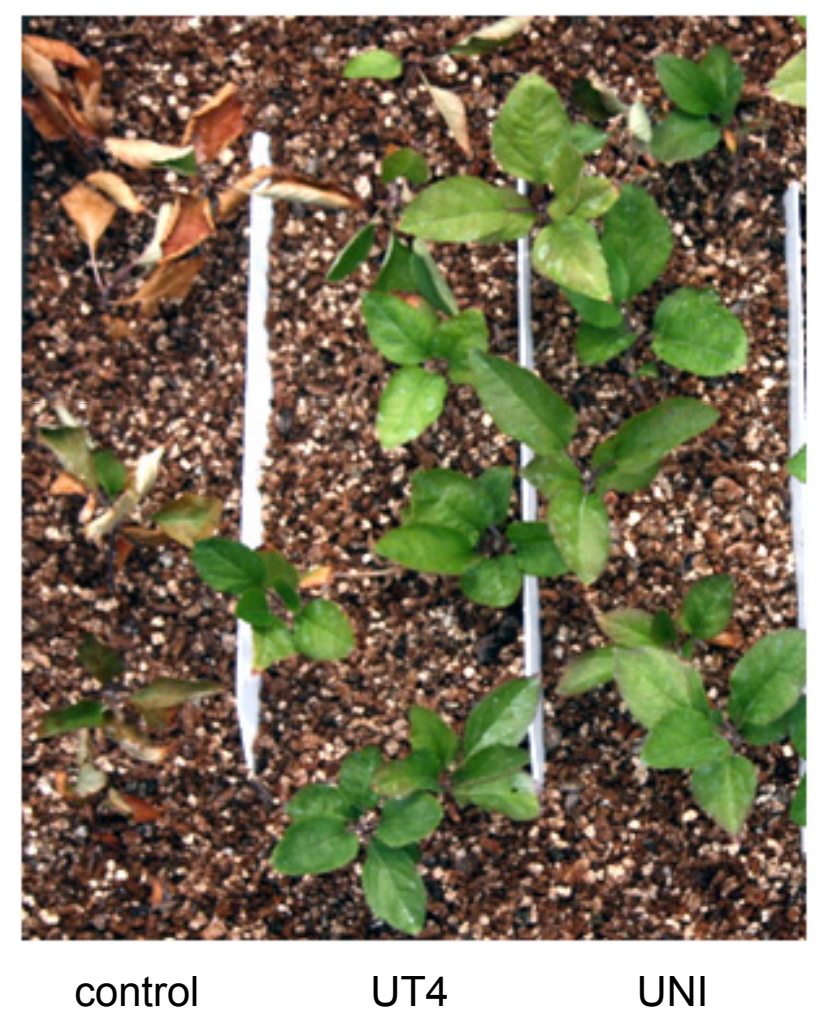

Figure 4 


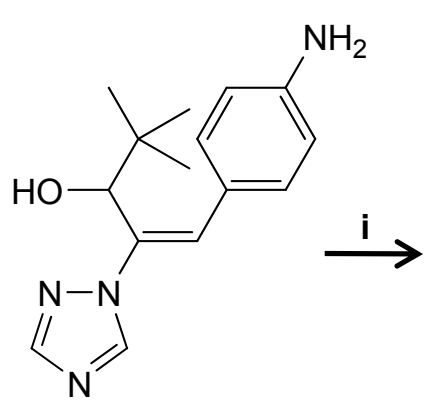

1

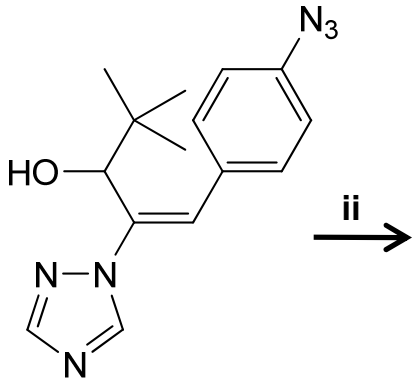

2

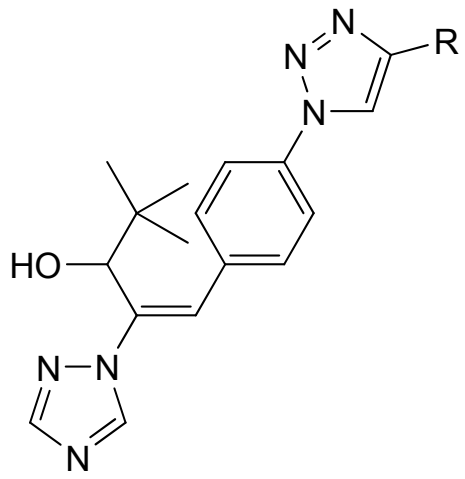

UT

Scheme 1 